\title{
Modeling the effects of a faint dust coma on asteroid spectra
}

\author{
Jorge Márcio Carvano $^{1}$ and Silvia Lorenz-Martins ${ }^{2}$ \\ ${ }^{1}$ Observatório Nacional (COAA), rua Gal. José Cristino 77, São Cristóvão, CEP20921-400 Rio \\ de Janeiro RJ, Brazil. \\ email: carvano@on.br \\ ${ }^{2}$ Universidade Federal do Rio de Janeiro/Observatório do Valongo. Lad.Pedro Antônio, \\ 43 - 20080-090 Rio de Janeiro, Brazil
}

\begin{abstract}
In this work we use a simple model to study the influence of a faint dust coma on asteroid spectra, in an effort to reproduce the unusual spectral behavior seen on the asteroid (5201) Ferraz-Mello and other objects.
\end{abstract}

Keywords. minor planets, asteroids

\section{Introduction}

Several lines of evidence suggest the possibility of a link between comets and asteroids: (a) the existence of icy objects, such as the trans- Neptunians and Centaurs, that are unlikely to develop a coma, since they never come close enough to the Sun; (b) the discovery that sublimation can stop on comets, either by the depletion of the volatile material or by the growth of a surface crust of refractory material; (c) the observation of objects in the main belt of asteroids that present temporal burst of activity, such as (7968) Elst-Pizarro, P/2005 U1 (Read) and (118401) 1999 RE70; (d) the association of meteor showers to asteroids, such as (3200) Phaeton and others; (e) the presence of asteroids with no visible coma in orbits with Tisserand parameter smaller than 3 .

Recently, Carvano et al. (2008) observed the $g, r, i, z$ colors of the asteroid (5201) Ferraz-Mello, whose dynamical behaviour strongly suggested it to be a captured Jupiter family comet. The colors were transformed to reflectance and comparison with a sample of similarly derived spectra of asteroids, Centaurs, TNOs and cometary nuclei revealed that reflectance spectrum of (5201) Ferraz-Mello is unusual (Fig. 1a), with a steep spectral gradient that is comparable to TNOs and Centaurs, but with an increase in the reflectance in the $g$ band that is not common in those populations. A similar behaviour is however seen in cometary nuclei that were observed in the presence of a faint dust coma (Fig. 1b). This suggests that the presence of a (unseen) faint dust coma might be the culprit of that peculiar increase in the reflectance in the $\mathrm{g}$ band.

In this work we use radiative transfer models in order test that hypothesis. We assume a spherical asteroid surrounded by a spherically symmetric dust coma. The reflectance of the asteroid is modelled using Hapke theory (Hapke (1993)), while the scattering due to the dust is modelled via a Monte-Carlo method.

\section{Model}

In a first attempt to model the effects of a faint dust coma to the reflectance spectrum of an asteroid we assume that the observed spectrum is the sum of two components: the Sun light reflected at the surface of the asteroid, attenuated as it traverses the coma in 

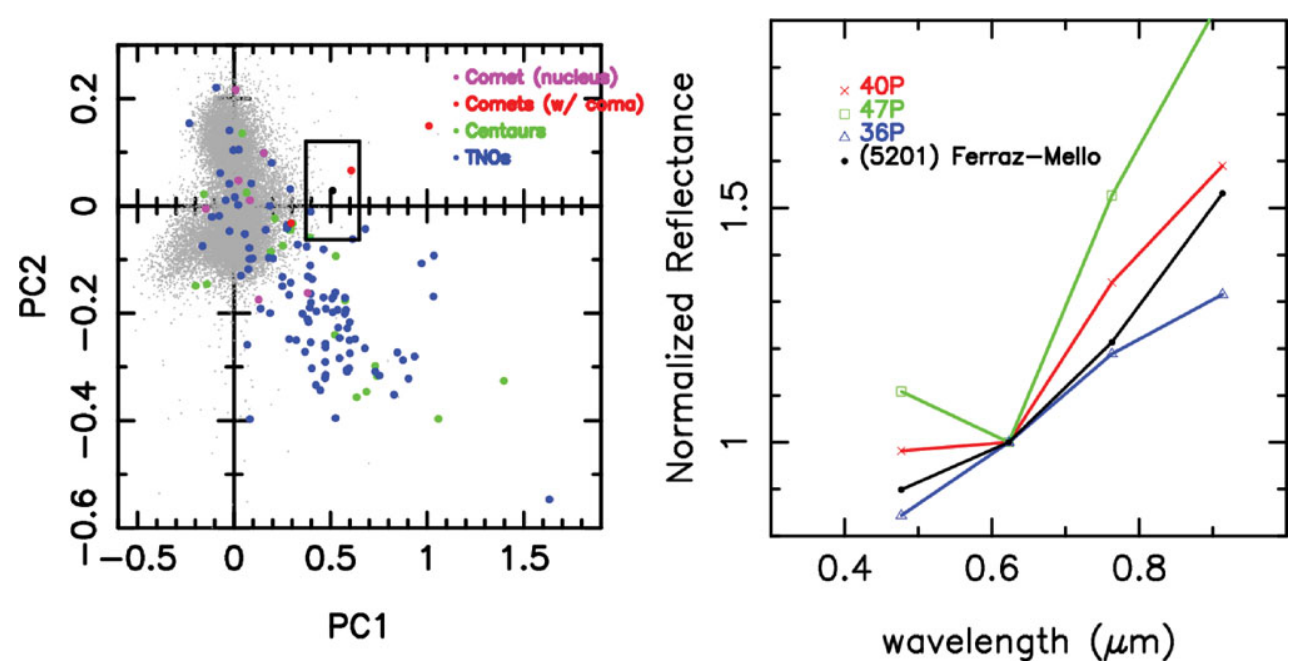

Figure 1. SDSS spectrum of (5201) Ferraz-Mello, compared with spectra of asteroids, Centaurs, TNOs and cometary Nuclei. (a) Principal component plot for (5201) Ferraz-Mello - black dot, uncertainties denoted by a box, asteroids - gray dots, Centaurs, TNOs, and cometary nuclei. Asteroid colors were obtained from the SDSS MOC3 (Gunn (1999)), TNOs and Centaurs from Neese (2006) and cometary nuclei from Snodgrass et al. (2007). Figure shows that (5201) Ferraz-Mello plots outside the main trend of small Solar System objects. (b) discrete spectra of (5201) Ferraz-Melo together with spectra of comet nuclei obtained in the presence of a faint dust coma.

its way in from the Sun and out towards the observer; and the Sun light that is scattered by the coma into the line-of sight of the observer.

To model the first component we consider a spherical asteroid and assume that the reflectance at its surface is described by Hapke's model (Hapke (1993)), disregarding the opposition effect and the macroscopic roughness of the surface. Therefore, the reflectance of each surface element of the asteroid is described by:

$$
R_{\lambda}=\frac{w_{\lambda}}{4 \pi} \frac{\mu_{0}}{\mu_{0}+\mu_{e}}\left[p(\theta)+H\left(\mu_{0}\right) H\left(\mu_{e}\right)-1\right]
$$

where $w(\lambda)$ is the single scatter albedo at a given wavelength, $p$ is the volumetric phase function (assumed to be a one-lobed Heyney-Greenstein function), $m u_{0}$ and $m u_{e}$ are, respectively, the cosines of the incidence and emission angles and $H$ is the Chandrasekar function and $\theta$ is the solar phase angle. The single scatter albedo is calculated from optical constants for a given grain sized $d_{A}$, using a geometric optics approximation. To compute the fraction of incident Sun light reflected at the surface of the asteroid we then consider a sphere made up of triangular facets and sum up the contribution of the reflectance of every facet that is visible and illuminated.

To model the contribution of the coma we consider a spherical cloud composed of spherical homogeneous particles whose volumetric density fall with the inverse of the square of the distance to the center of the asteroid and use a Monte Carlo method to calculate the amount o radiation scattered towards the observer, assuming single scattering. The code essentially shoots a energy parcel at a random position on the cloud, picks a random optical depth for the interaction and then calculates the amount of energy that is removed by extinction and the amount that is scattered in the direction 
of the observer. For a individual parcel the fraction of energy that is sent towards the observer is

$$
S_{i \lambda}=Q_{S_{\lambda}} \pi d_{c}^{2} \frac{N_{0}}{r_{i}^{2}} p_{\lambda}(\theta) e^{-2 \tau_{i \lambda}}
$$

where $N_{0}$ is the number of particles, $d_{C}$ is the diameter of the particles in the coma, $Q_{S_{\lambda}}$ and $p_{\lambda}(\theta)$ are, respectively, the scattering efficience and phase function of the grains, calculated using Mie theory from optical constants for a given grain size.

The total reflectance from the asteroid and the coma is then calculated as

$$
E_{\lambda}=R_{\lambda} e^{-2 \tau_{\lambda}}+M^{-1}\left(\frac{r_{C}}{r_{A}}\right)^{2} \sum_{i}^{M} S_{i \lambda}
$$

where $r_{C}$ and $r_{A}$ are respectively the radius of the coma and of the asteroid and $M$ is the number of parcels shot at the cloud.

Finally, in order to simulate a material with spectral behaviour akin to outer Main Belt asteroids we follow Grundy (2009) to derive optical constants for a mixture of water ice and tholins using a Maxwell-Garnet model; the optical constants for the endmembers were taken from Khare et al. (1984) and G. Hansen (personal communication). The same optical constants were used for the grains in the coma and on the asteroid surface.

\section{Preliminary results and perspectives}

The resulting model has 5 free parameters: $N_{0}, d_{C}, d_{A}$, the coefficient $\psi$ of the HeyneyGreenstein function used in 2.1 and $\eta$, the volumetric fraction of tholins in the grains used in the Maxwell-Garnet model. At this stage we consider only the case where the asteroid is observed at zero phase angle - the lack of opposition coefficients in 2.1 is justified since we are interested here in the spectral behaviour of the reflected light, and the opposition effect is independent of wavelength. Likewise, since a gray phase function for the surface is assumed, we use an arbitrary value of $\psi=0.1$ for the Heyney-Greenstein coefficient.

After some experimentation, we adopted $\eta=0.05$. From the remaining parameters, $N_{0}$ and $d_{C}$ have the most influence of the resulting spectra. Numerical tests show that a spectral behaviour similar to the objects discussed in section 1 can be reproduced using sub-micron grains in the coma. Figures 2a-c show the effect of grains with $d_{C}=0.2 \mu \mathrm{m}$ for increasing values of $N_{0}$, and the resulting SDSS spectra for the highest $N_{0}$ is shown in Figure 2d.

It is clear that the simple model used here is able to produce an increase in the reflectance in the shorter wavelengths, as expected, but the resulting spectra tend to be bluer than the asteroid spectrum without the coma.

The results presented here suggest that the presence of a faint coma composed of submicron particles can produce the unusual reflectance spectra that is observerd in (5201) Ferraz-Mello and other objects. To fully understand the problem however it is necessary a more accurate model, that includes multiple scattering in the coma, a size distribution for the grains and possibly a two component coma. It is also desirable to study the effects of the solar phase angle of the observations on the reflectance spectra of the objects. 

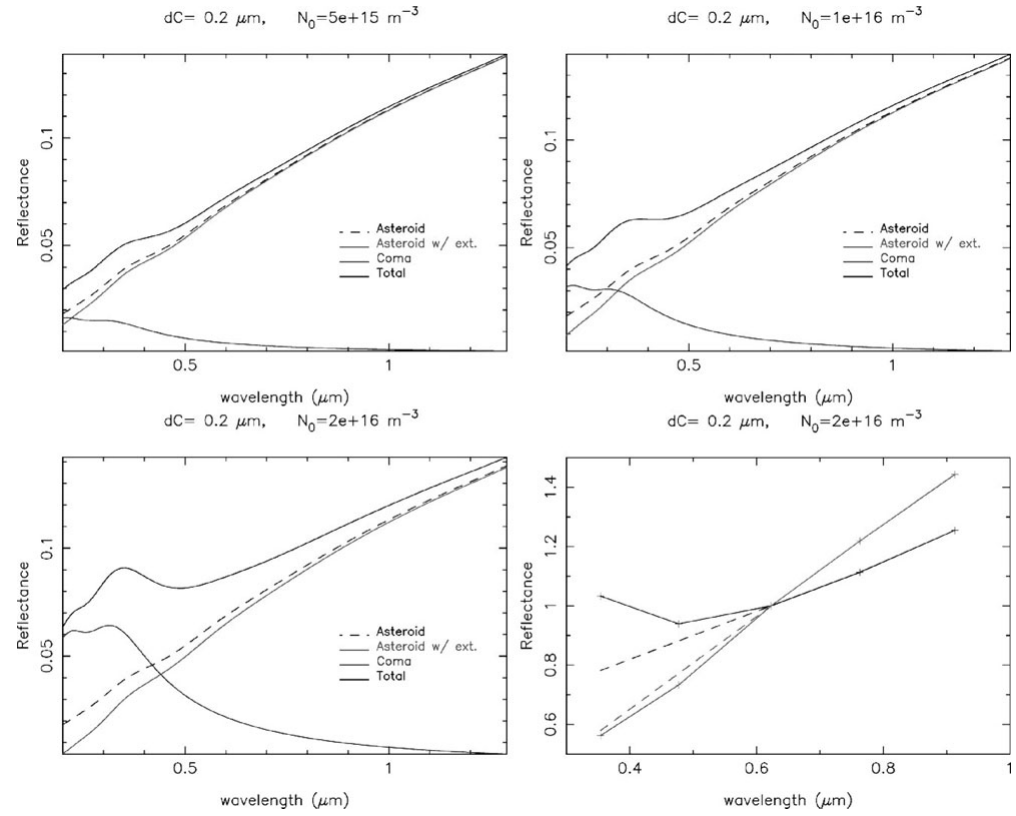

Figure 2. (a-c) Resulting spectra for increasing dust density in the coma, with $d_{C}=0.2 \mu \mathrm{m}$, $d_{A}=10 \mu \mathrm{m}$. (d) Convolution of the model spectra with the SDSS bandpasses without a coma and with the same model as in (c); the dotted lines are extensions towards the $u$ filter of the spectral inclination between the $r$ and $i$ filters.

\section{References}

Carvano, J. M., Ferraz-Mello, S., \& Lazzaro, D. 2008, A\& A, 489, 811

Grundy, W. M. 2009, Icarus, 199, 560

Gunn, J. E. 1999, Bulletin of the American Astronomical Society, 31, 1418

Hapke, B. 1993, Theory of reflectance and emittance spectroscopy, (Cambridge: Cambridge University Press)

Khare, B. N., Sagan, C., Arakawa, E. T., Suits, F., Callcott, T. A., \& Williams, M. W. 1984, Icarus, 60,127

Neese, C. 2006, NASA Planetary Data System, EAR-A-COMPIL-3-TNO-CEN-COLOR-V3.0, 57

Snodgrass, C., Lowry, S. C., \& Fitzsimmons, A. 2007, ArXiv e-prints, 712 